\title{
Comparative Studies on the Ultrastructure of Malignant Melanoma in Fish and Human by Freeze-etching and Transmission Electron Microscopy
}

\author{
Rüdiger Riehl ${ }^{1},{ }^{3}$ Manfred Schartl ${ }^{2}$, and Gerhard Kollinger ${ }^{2}$ \\ ${ }^{1}$ Forschungsgruppe Dermatologie der Universitäts-Hautklinik Heidelberg, 6900 Heidelberg, Federal Republic of Germany \\ ${ }^{2}$ Genetisches Institut der Justus Liebig-Universität Gießen, Federal Republic of Germany
}

Summary. Malignant melanomas (MM) in the fish $X i$ phophorus and in humans were studied both by transmission electron microscopy (TEM) and freeze-etching (FE). In both fish and human melanomas the cells show interdigitations of the plasma membranes. The nuclei are large and lobulated and have many nuclear pores. Melanosomes are abundant and melanosome complexes ("compound melanosomes") occur regularly. Pinocytotic vesicles could be demonstrated in fish and human melanomas showing local differences in frequency and distribution patterns in the tumor. Intercellular junctions are lacking in MM cells from fish and humans. The FE technique showed considerable advantages in demonstrating membrane-surface peculiarities such as nuclear pores or pinocytotic vesicles. The FE replicas of fish melanomas are like those of humans. These findings may support the hypothesis that melanoma in fish and humans reflect the same biological phenomenon.

Key words: Malignant melanoma - Fish - Human Freeze-etching - Transmission electron microscopy

\section{Introduction}

The Xiphophorus melanoma system has been shown to be enormously useful as a model for the study of the etiology of neoplasia in general (Anders et al. 1981). It makes it possible to reduce the complexity of cancer and cancer-related phenomena to a few biological elements involved in tumor formation. The development of neoplasia requires an oncogene, which, after impairment, deletion or elimination of its regulatory genes is able to mediate neoplastic transformation in Xiphophorus (for a recent review see Anders et al., to be pub-

3 Present address and offprint requests to: Dr. Rūdiger Riehl, Institut für Zoologie II der Universität Düsseldorf, Universitätsstr. 1, D-4000 Düsseidorf, Federal Republic of Germany lished). As it is generally accepted that there is a common principle underlying neoplasia in all multicellular animals, studies on melanoma formation in the experimental system Xiphophorus can produce results that are impossible to gain from clinical studies on humans, but are useful for the understanding of melanoma formation in man. To evaluate the data and the conclusions from the work on Xiphophorus, we tried to compare the melanomas from fish and humans on all levels of biological organization.

The morphology of fish melanoma has been successfully investigated by light microscopy and transmission electron microscopy (Weissenfels et al. 1970; Vielkind et al. 1971 a, b; Sobel et al. 1975), while freezeetch studies (FE), which have been very useful in investigating the ultrastructure of normal and neoplastically transformed pigment cells in humans (Breathnach 1973; Breathnach et al. 1973; Lea et al. 1976; Tilgen et al. 1981; Riehl and Tilgen 1982) were still lacking.

The present study was carried out to compare the ultrastructure of melanoma in fish and humans by means of TEM and FE, and to obtain a better understanding of the ultrastructural changes during tumor formation.

\section{Materials and Methods}

\section{Materials}

Fish Melanoma. Melanotic melanoma bearing Xiphophorus hybrids $(n=5)$ were kindly provided by Dr. A. Anders, Giessen. They were produced by crossing Xiphophorus maculatus (population Rio Jamapa) carrying the gene complex $S d-T u$ with Xiphophorus helleri (population Belize River) lacking this gene complex; the resulting $F_{1}$ hybrids were backcrossed to $X$.helleri. For a detailed description of this crossing and the underlying principles of melanoma formation in $X i$ phophorus see Anders and Anders (1978). Only fish carrying malignant melanoma (for classification see Anders et al. 1979) were used in this study.

Human Melanoma. Human malignant melanotic melanomas $(n=4)$ were provided by courtesy of Prof. E. Paul, Center for Dermatology and Andrology, Skin Clinic Giessen 


\section{Methods}

Light Microscopy

All specimens were fixed in Bouin's solution. Excess picric acid was eluated with $70 \%$ ethanol. The fixed specimens were dehydrated and embedded in paraffin; $5-\mu \mathrm{m}$ sections were cut with a Leit $z$ base sledge microtome and stained with acid alizarine blue and aniline blue orange $G$ in a modification of the azan staining method (see Romeis 1968).

\section{Transmission Electron Microscopy}

Fish Melanoma. Small pieces of melanoma tissue were fixed in $4 \%$ glutaraldehyde for $3 \mathrm{~h}$. After washing in phosphate-buffered saline with the osmolality of fish cells, the specimens were postfixed in $2 \%$ osmic acid. The tissue blocks were prestained with $2 \%$ uranyl acetate in $20 \%$ ethanol at $60^{\circ} \mathrm{C}$ for $3 \mathrm{~h}$, then they were dehydrated and embedded in ERL-4206. Ultrathin sections were cut with a diamond knife using a Reichert ultramicrotome Om U 3 and examined in a Zeiss EM $10 \mathrm{~A}$ electron microscope.

Human Melanoma. Tissue pieces were taken from freshly excised melanomas and prepared according to Peracchia and Mittler (1972) by fixation with $3 \%$ glutaraldehyde in $0.1 \mathrm{M}$ sodium cacodylate buffer ( $\mathrm{pH} 7.4)$ in addition to 6 drops of $\mathrm{H}_{2} \mathrm{O}_{2}(30 \%)$ for $3 \mathrm{~h}$. The pieces were washed several times, then passed for $2 \mathrm{~h}$ through glutaraldehyde ( $\mathrm{pH} 7.4$ ), washed again, and postfixed with $2 \%$ osmium tetroxide for $2 \mathrm{~h}$. Specimens were dehydrated and embedded in Epon 812. A Reichert ultramicrotome Om U 3 was used to cut ultrathin sections. These were double-stained with uranyl acetate and lead citrate (Reynolds 1963) and examined in a Philips EM 400 and a Zeiss EM $10 \mathrm{~A}$.

Freeze - etching; Fish and Human Melanoma

Small pieces of melanoma tissue were removed from fish anesthetized with Anestesin (4-aminobenzoic-acid-ethylester; Serva, Heidelberg) and fixed immediately in $30 \%$ glutaraldehyde for 1 to $3 \mathrm{~h}$ at $4{ }^{\circ} \mathrm{C}$. The buffer used in all experiments was $0.2 \mathrm{M}$ sodium veronal acetate, pH 7.3.

Freezing, fracturing, shadowing, and cleaning of the specimens was described in a previous paper (Riehl and Tilgen 1982). The cleaned replicas were picked up on standard 300-mesh copper grids; to ensure that the replicas adhere to the grid, grids were dipped in "grid blue" (Hudson et al. 1979). The replicas were examined in a Philips EM 400 and a Zeiss EM 10A electron microscope. The terminology for fracture faces is according to Branton et al. (1975).

\section{Results}

\section{Anatomic and Histologic Observations}

Fish. All melanomas from fish used for this study were heavy nodular cell masses with smooth regular surfaces with a lobulated appearence. As is characteristic of this genotype, melanoma formation starts at the age of 2-3 months in the region of the dorsal fin and the melanoma is composed of abnormal melanocytes and abnormal melanophores. The tumor spreads and finally covers almost one-third of the fish's surface (Fig. 1). Because of the three-dimensional growth of the melanoma, the fin tissue is replaced by the growing tumor tissue as neoplasia development proceeds. In histologic sections, the tumor appears as a well vascularized pigmented thickening of the corium with pigmented cells infiltrating the thickened epidermis and the muscular tissue by invading the myosepta (Fig. 2).
Following the complete infiltration of the connective tissue, the muscle fibers degenerate and are replaced by transformed melanocytes and/or melanophores. The transformed pigment cells of fish malignant melanoma are larger than normal ones. They are round or polygonal and some cells show dendritic processes that interlace with each other. The nuclei are polymorphic. All other subcellular structures are obscured by the dense melanin pigmentation.

\section{Transmission Electron Microscope Observations}

Fish. The ultrastructural morphology of malignant melanoma of fish shows that the tumor is composed of transformed pigment cells. The melanoma cells are tightly packed and show dendritic processes. The malignant melanoma consists of a large number of incompletely differentiated cells and a smaller number of well differentiated cells (Fig.3). The well differentiated transformed pigment cell is highly dendritic (Fig.4). The interdigitating membranes of the dendritic processes form a meshwork of cells resembling the nerve tissue cells. Intercellular junctions are not present.

The nuclei of the transformed pigment cells are large and lobulated exhibiting protrusions, pockets, and numerous nuclear pores (Fig. 6). There is strong pinocytotic activity in the melanoma cells as may be seen by the numerous pinocytotic vesicles (Fig.6), which are mostly arranged in an irregular distribution pattern; patch-like areas are very rare.

The spheroidal or ellipsoidal melanosomes (diameter 200-650 nm) are distributed throughout the cellular dendrites, whereas the perinuclear cytoplasm is free of melanosomes. They are monomorphous, mostly rounded, and variable size. The degree of melanization of melanosomes correlates positively with the degree of cell differentiation. Highly differentiated cells are heavily melanized concealing the internal structure and occasionally the surrounding membrane (Fig. 10). In less differentiated cells the melanosomes are incompletely melanized (Fig. 11) and no internal architecture (protein layer) or higher order of organization of melanin granules is detectable.

Human. In accordance with the TEM investigation on fish melanoma, we have found that the human melanoma is formed by abnormal melanocytes with enlarged nuclei. Cells resembling melanophores, which are present in the fish, are not present in human melanoma. There are melanoma cells with different degrees of differentiation and maturation. The human melanoma cells are tightly packed and may exhibit prominent dendritic processes (Fig. 13). The nuclei of the enlarged cells were frequently bizarre and hyperplastic. In some nuclei, the nucleonema was a compact mass, in others it was dispersed as strands. Many of the 

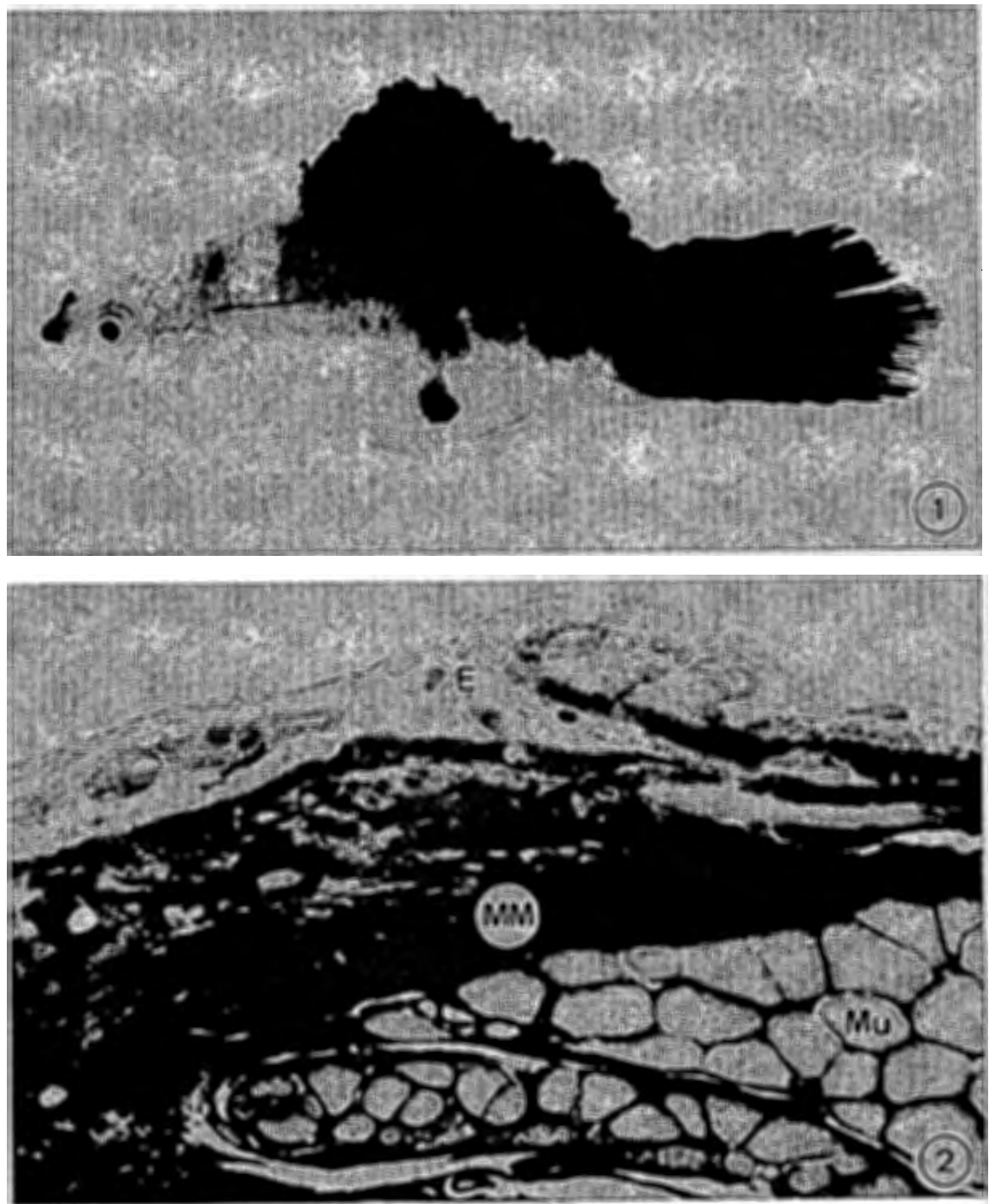

Fig.1. Pigmented platyfishswordtail hybrid bearing a melanotic malignant melanoma

\author{
Fig. 2. Histologic section \\ through melanomatous skin \\ of Xiphophorus showing \\ hyperplastic epidermis $(E)$, \\ pigmented thickening of the \\ corium, and infiltration of \\ muscular tissue $(M u)$ by \\ melanoma cells $(\mathrm{MM}) \times 1,100$
}

melanoma cells contain a prominent Golgi apparatus and numerous mitochondria.

The most impressive feature of the pigmented melanoma cells was the occurrence of numerous, mostly spheroidal melanosomes with a diameter ranging between 200 and $500 \mathrm{~nm}$. Some of the melanosomes showed ellipsoidal profiles and melanosomes showing a granular structure were common (Fig. 14), whereas lamellar melanosomes were seen less frequently (Fig. 14, arrow).

\section{Freeze-etching Observations}

Fish. In accordance with the TEM investigation, FE replicas show tightly packed melanoma cells that are highly dendritic (Fig. 5). There are many interdigitations of their plasma membranes and the nuclei are large, exhibiting bizarre, lobulated surfaces. Protrusions, nuclear pockets and numerous nuclear pores were found regularly (Figs. 7, 8). Strong pinocytotic activity is shown by abundant pinocy totic vesicles, which were mostly arranged in irregular patterns (Fig. 9). In all FE replicas intercellular junctions were lacking.

Numerous vesicular structures were distributed throughout the cytoplasm. Taking into account the frequency of melanosomes in TEM, it may be stated that most of these vesicles are melanosomes. The melanosomes exhibit three types of fracture face in FE replicas: types 1 and 2 represent complimentary fracture faces of the limiting membrane ( $P$ lasma und External face) and type 3 shows a cross fracture of the melanosome. Types 1 and 2 are more frequent than type 3 . The membrane surrounding the melanosomes 


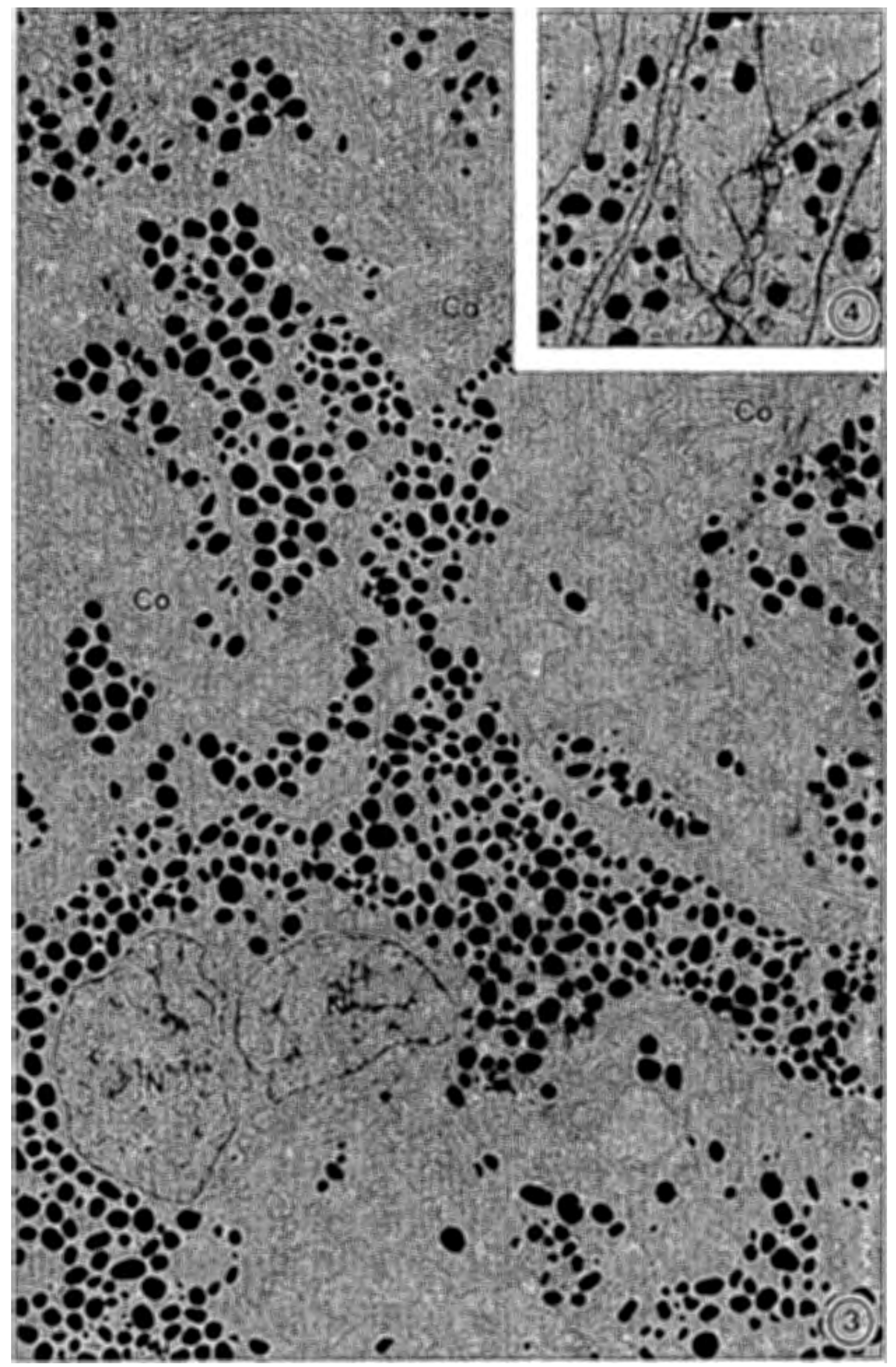

Fig. 4. Meshwork of

interdigitating dendritic processes (TEM, fish)

$\times 18,000$
Fig.3. Low-power electron micrograph of a typical area of $M M$ in fish. The cells show many interdigitating dendritic processes. The cytoplasm is filled with abundant

melanosomes. $\mathrm{Co}=$ collagen; $N=$ nucleus. $\times 7,000$ 


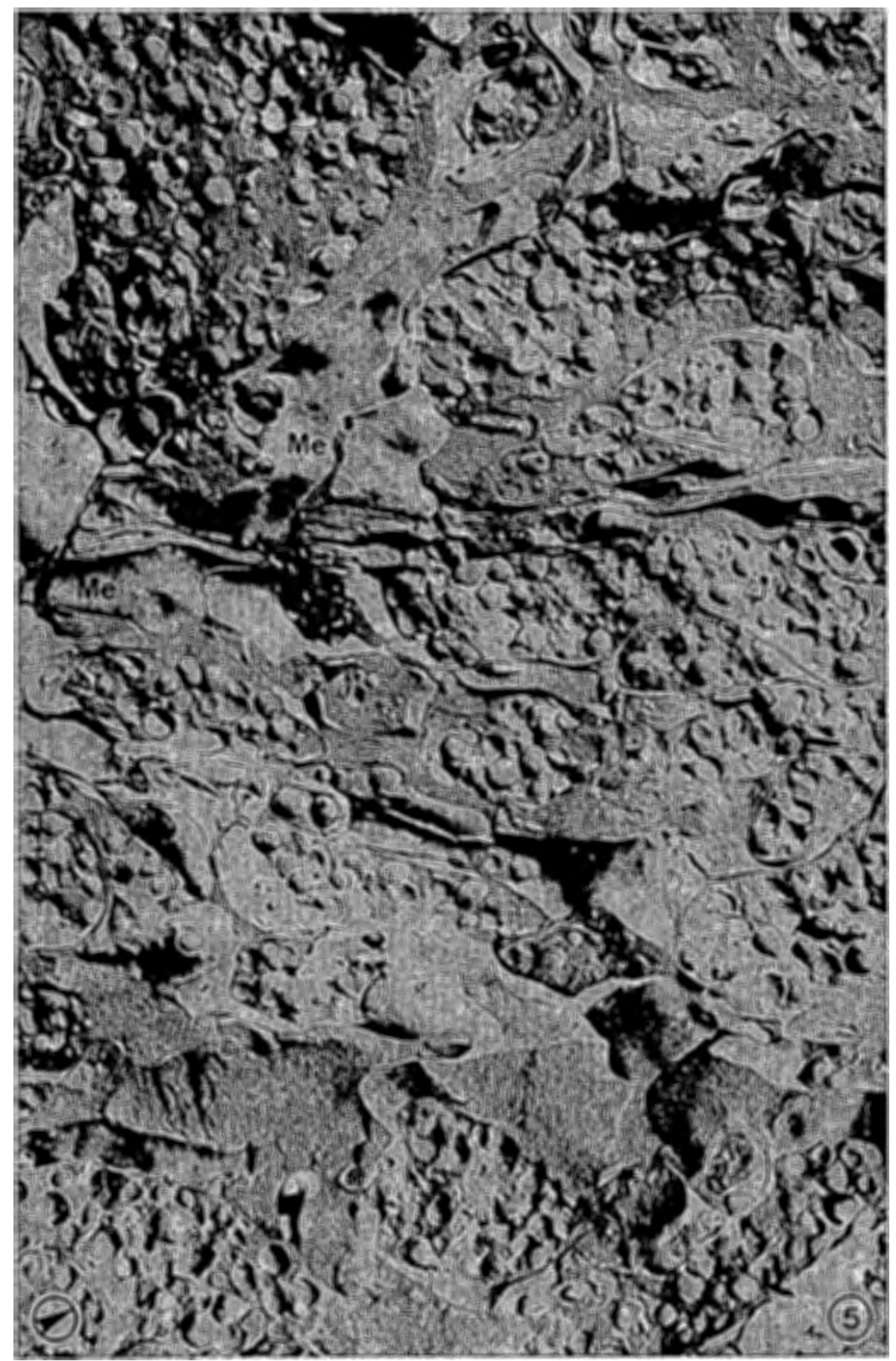

Fig. 5. Low-power freeze-etch micrograph of MM in fish. Note the interdigitations of cell membranes $(\mathrm{Me})$ and the abundant melanosomes. Arrowhead indicates the shadowing direction. $\times 9,600$ 

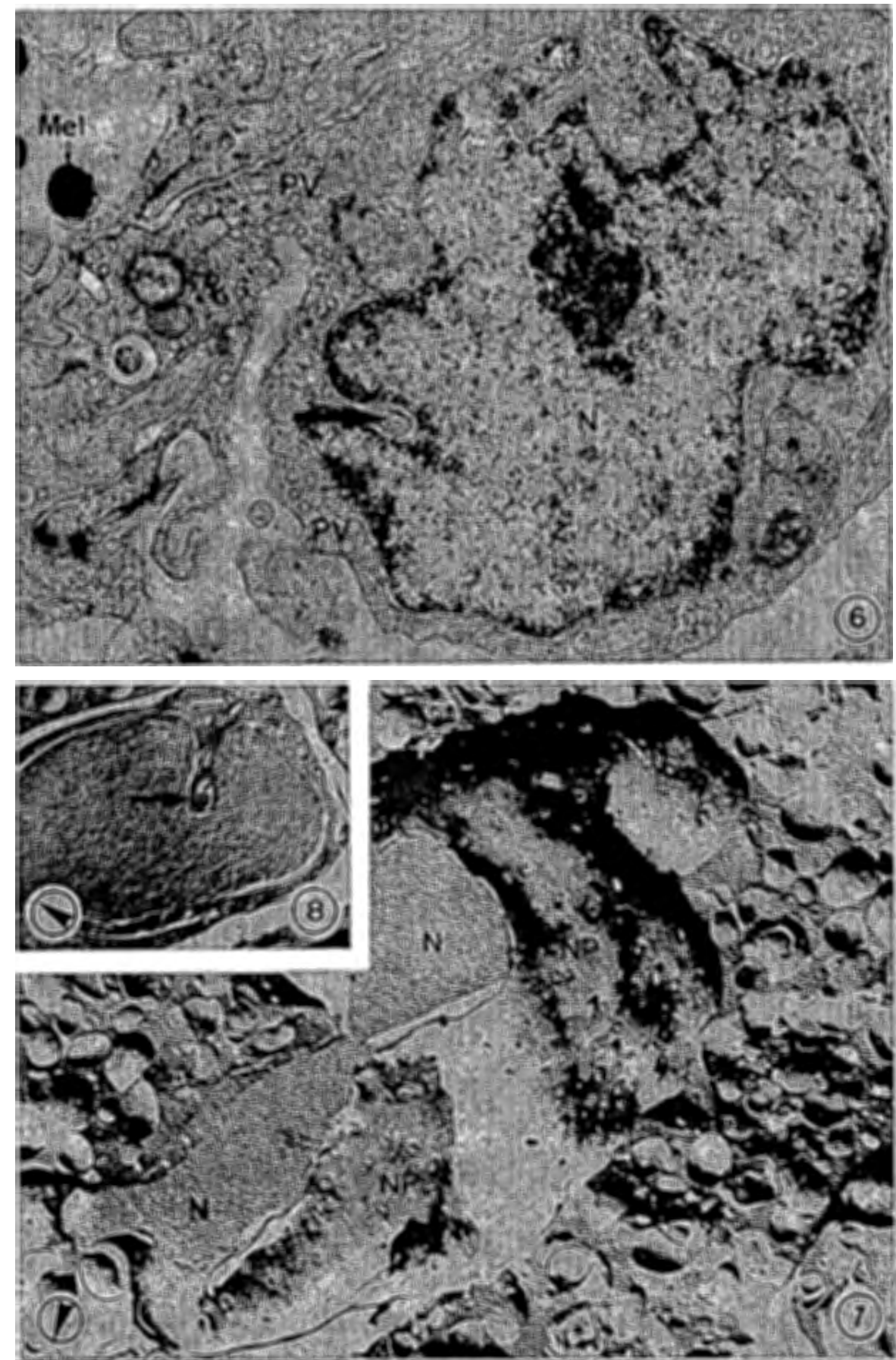

Fig. 6. TEM micrograph of a lobulated nucleus $(N)$ of a fish MM cell with a nuclear pocket (arrow). In the vicinity of the cell membrane a lot of pinocytotic vesicles $(P V)$ occur. $\mathrm{Mel}=$ melanosome. $\times 22,800$

Fig. 7. FE micrograph of a nucleus $(N)$ in fish MM. Onc part of the nucleus shows its surface with nuclear pores $(N P)$, whereas the other part shows its interior resulting from a cross fracture. $\times 24,300$

Fig. 8. FE micrograph of a fish MM nucleus with a nuclear pocket (arrow). Arrowheads indicate the shadowing direction. $\times 13,500$ 


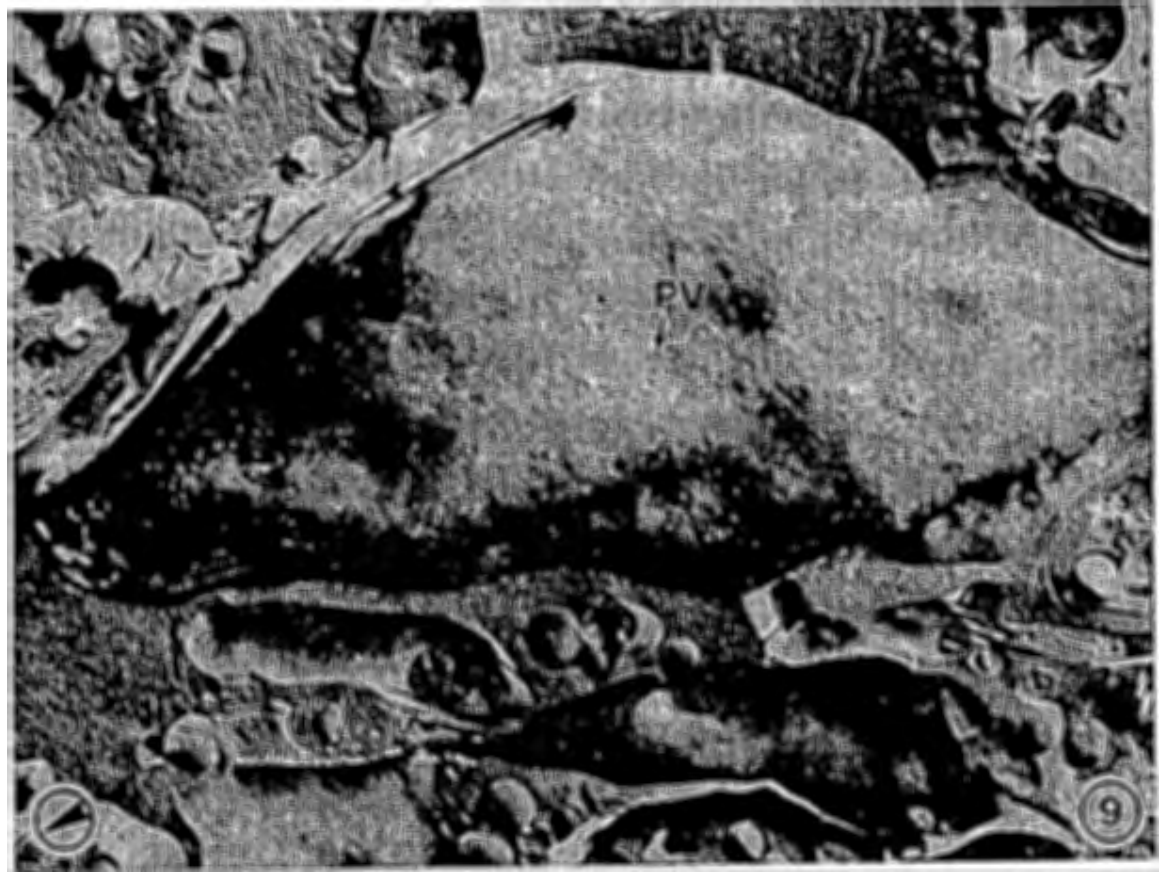

Fig.9. Area of a fractured membrane with numerous pinocytotic vesicles $(P V)$ (Freeze-etch micrograph, fish). Arrowhead $=$ shadowing direction. $\times 13,800$ carries particles with a random distribution. Occasionally melanosome complexes ("compound melanosomes") are present consisting of from only a few to numerous melanosomes that are all enveloped by a separate membrane (Fig. 12).

Human. As in the TEM investigations, freeze-etching exhibits melanoma cells with prominent dendritic processes and distinct interdigitations of their plasma membranes (Fig. 15); desmosomes are lacking. It clearly shows the hyperplastic nuclei with numerous nuclear pores (Fig. 15). Membrane surfaces have abundant pinocytotic vesicles, which are mostly arranged in irregular distribution patterns (Fig. 16).

Freeze-etching of melanosomes clearly exhibits a double-layered membrane (Fig. 17). The highly ordered internal arrangement found in immature melanosomes by TEM is not detectable in FE replicas. Fracture faces of the limiting membrane carry isolated particles that are not distributed in any particular pattern. These particles are not seen in TEM micrographs. Melanosome complexes also occur in humans.

\section{Discussion}

The aim of the present study was to find out what differences and similarities there are on the histologic and ultrastructural level between Xiphophorus melanoma and human melanoma. Both tumors showed extensive conformity in gross morphology with their strong pigmentation, lobulated nuclei, pinocytotic activity, and inter- digitation of dendritic cell processes. A further common feature of fish and human melanoma cells is the occurrence of melanosome complexes. In fish melanoma, however, the interdigitations of the highly differentiated cells are more prominent than in any human melanoma cells. Furthermore, mature melanosomes of fish melanoma cells show no internal structure, whereas the majority of melanosomes of human melanoma cells show mostly a granular or sometimes a lamellar internal architecture (compare Hunter et al. 1978). In fish cells, coarse-grained arrangement of melanin was found only in the immature premelanosomes. These ultrastructural differences between fish and human melanoma may be due to the presence of an additional cell type in fish melanoma, the melanophore. Melanophores have been shown to be the terminal stage of pigment cell differentiation in fish (Anders et al. 1979). In the nontumorous state, the highly dendritic melanophore has a specialized function in color change in lower vertebrates. In mammals, pigment-cell differentiation ends in the nontumorous as well as in the tumorous state at the preceding stage, the melanocyte.

Different observations have been made with regard to the presence of cell junctions in fish and human melanoma. While a lack of intercellular junctions was reported for human melanoma (Zelickson 1967; Breathnach 1971; Riehl and Tilgen 1982) and fish melanoma (Vielkind et al. $1971 \mathrm{a}$; Sobel et al. 1975) these authors, who have already compared the ultra- 

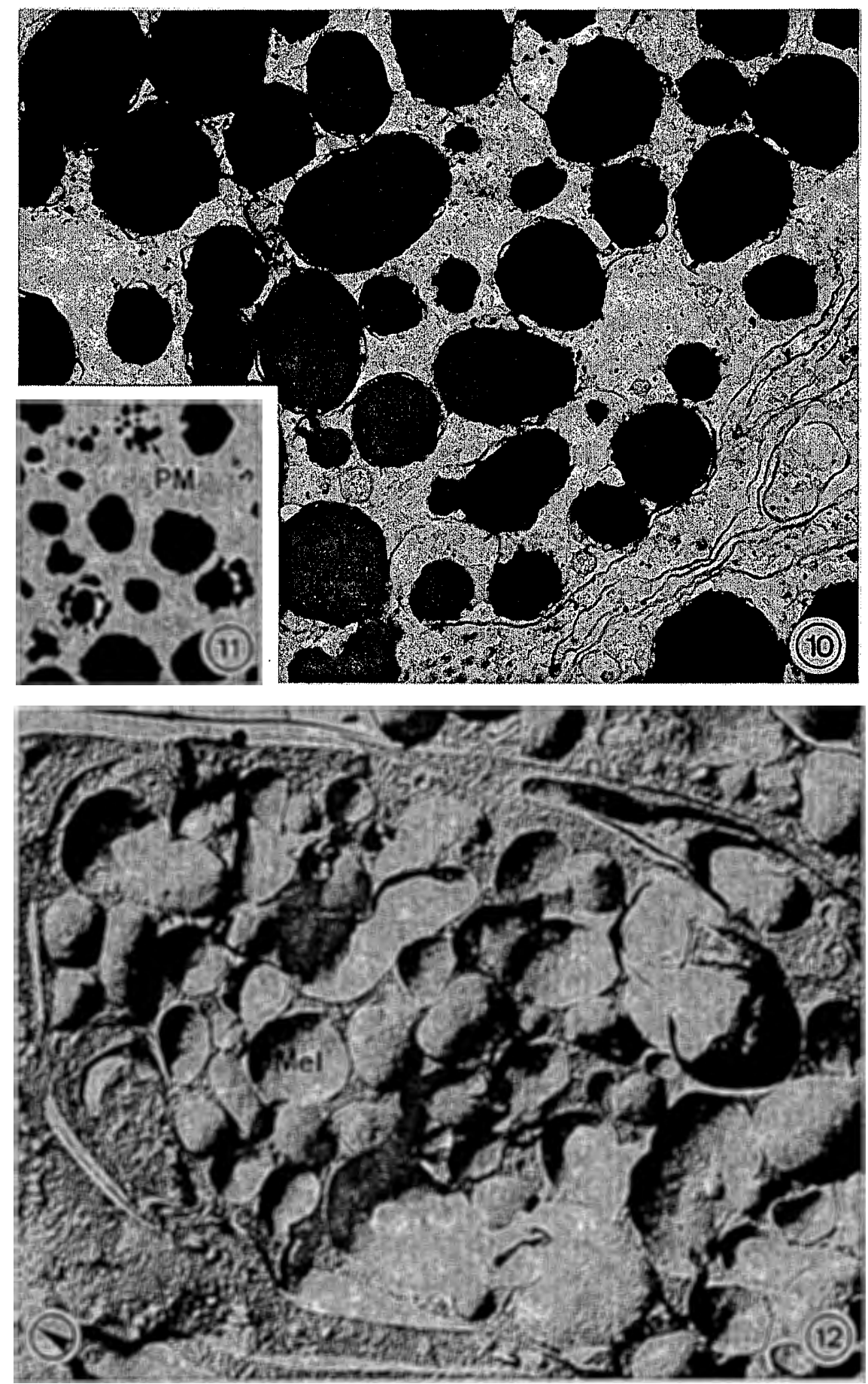

Fig.10. TEM high-power micrograph of mature melanosomes from a platyswordtail hybrid. $\times 38,000$

Fig.11. TEM high-power micrograph of melanosomes and premelanosomes $(P M)$ with coarse-grained arrangement of the melanin Fish, $\times 40,000$
Fig. 12. FE micrograph of a melanosome complex

("compound melanosome") in a fish MM cell. The complex consists of from a few to numerous melanosomes $(\mathrm{Mel})$, which are enveloped by a separate membrane. Arrowhead = shadowing direction. $\times 36,500$ 


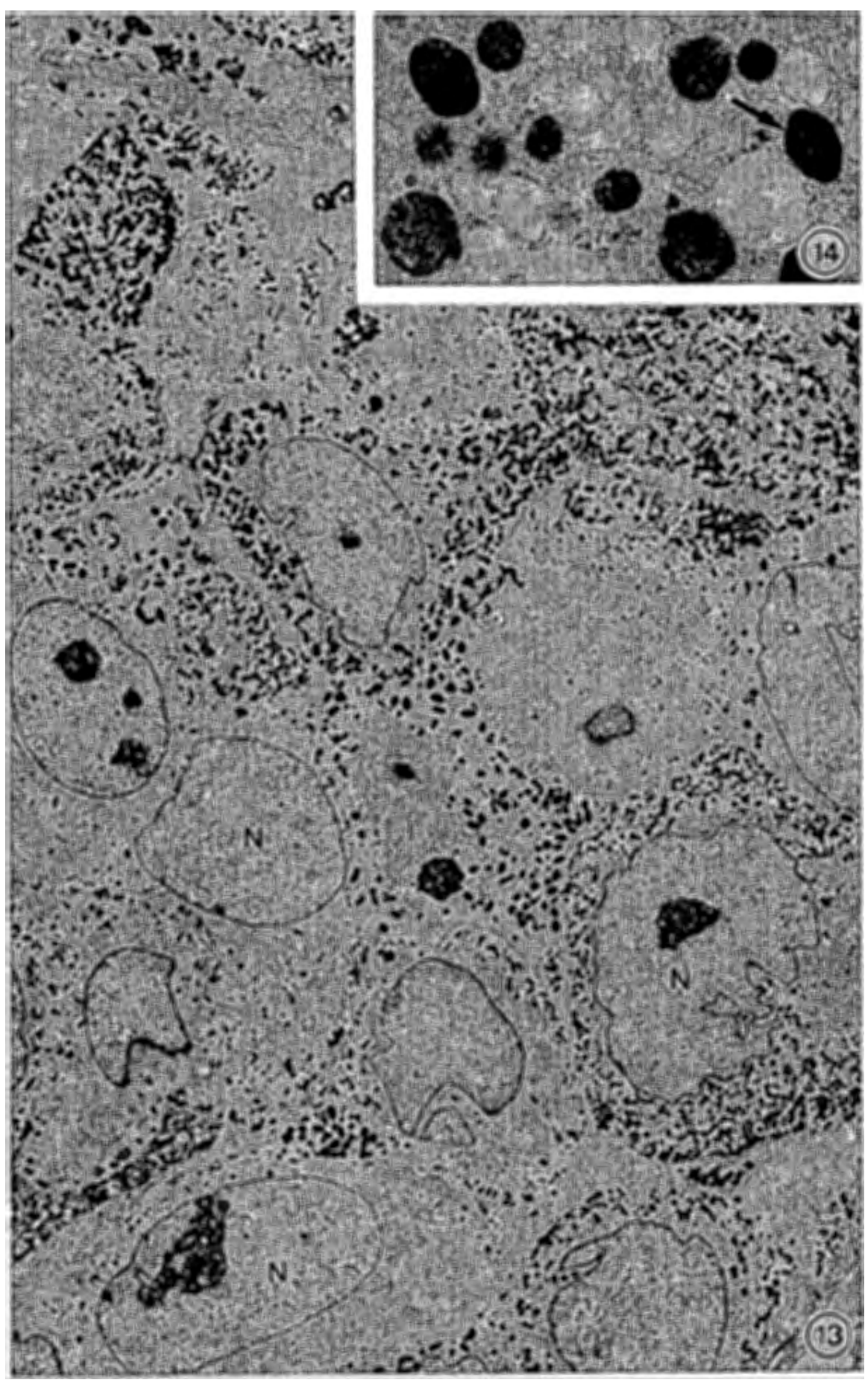

Fig. 14. High-power electron micrograph of melanosomes from a human MM. Most belong to the granular type. The arrow points to a lamellar melanosome. $\times 37,500$
Fig.13. Low-power micrograph (TEM) of a human MM. The nuclei $(N)$ are large and lobulated. The cells contain numerous melanosomes. $\times 3,300$ 

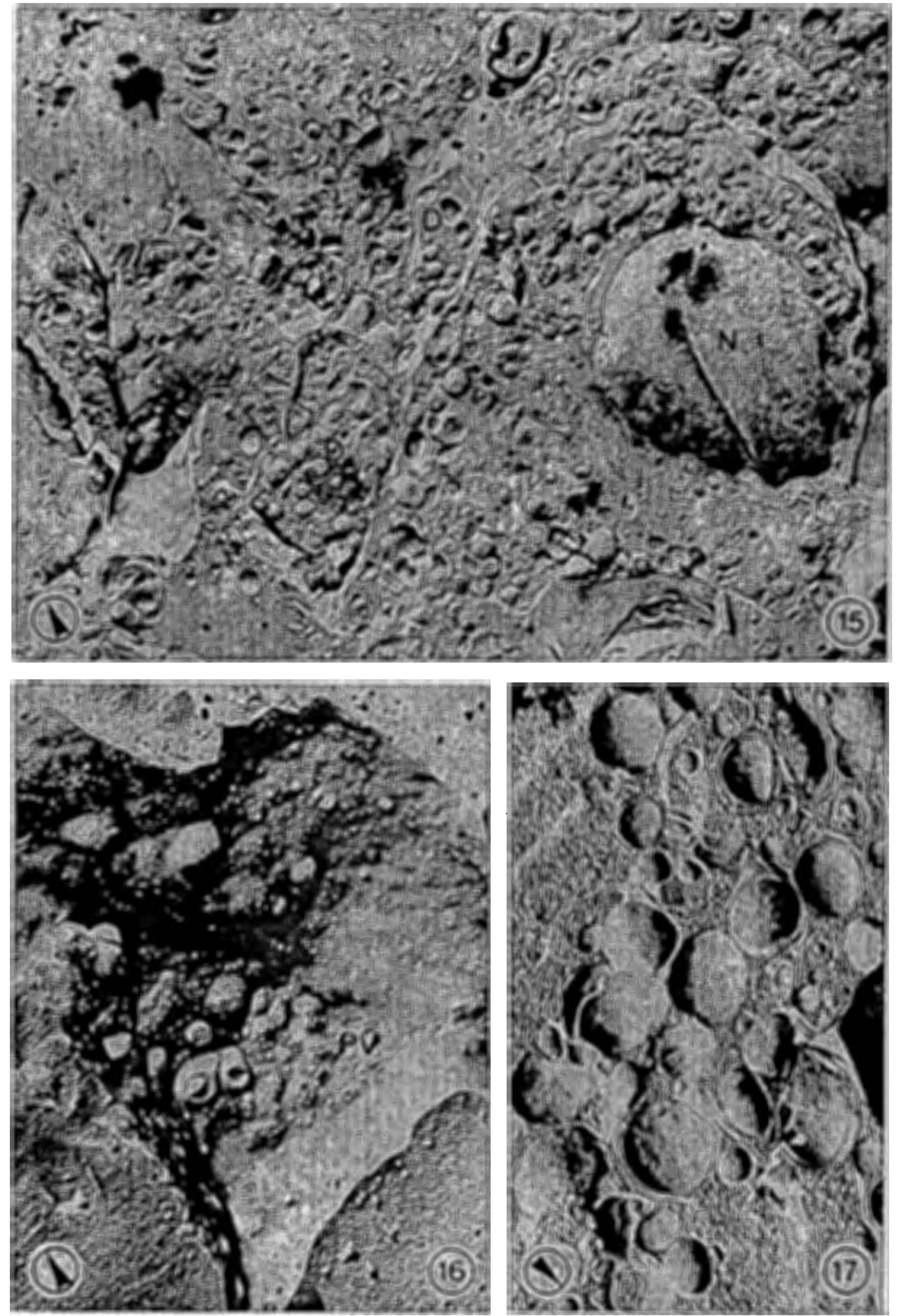

Fig. 15. Low-power FE micrograph of a human MM. The nucleus $(N)$ shows a bizarre surface with numerous nuclear pores. Note the dendritic processes $(D)$ of the melanoma cells, which are filled with many melanosomes. $\times 6,600$

Fig. 16. Area of a human MM with numerous pinocytotic vesicles $(P V)$ that are not arranged in a regular pattern. Freeze-etch micrograph. $\times 16,200$

Fig. 17. High-power micrograph of melanosomes showing a double-layered membrane; freeze-etching. Arrowheads indicate shadowing direction. $\times 30,400$ structure of fish and human melanoma on the TEM level, reported that junctional changes and desmosomes occur in human melanoma, but not in fish melanoma. Our study could not confirm the statement of Sobel et al. (1975). So far intercellular junctions have not been found in normal human melanocytes, melanocytes of nevi or melanomas, or in fish melanomas.
Freeze-etching gave additional information on surface characteristics. In FE replicas, the plasma membrane of Xiphophorus melanoma cells show abundant pinocytotic vesicles. This finding agrees with the TEM results of Weissenfels et al. (1970) and Sobel et al. (1975), who frequently found pinocytotic vesicles in the membranes of melanoma cells in fish. The abundance of pinocytotic vesicles points to strong transport activ- 
ity, which is believed to reflect the exocytosis of "basement-membrane-like" material in these areas (Sobel et al. 1975).

In FE replicas, it is difficult to distinguish melanosomes from other vesicular elements such as mitochondria or lysosomes in every case. According to the observations of Lea et al. (1976) on B16 melanoma and Riehl and Tilgen (1982) on human melanoma, this can only be performed by parallel studies of ultrathin sections of the same melanoma.

Melanosome complexes, which have been shown by FE to occur regularly in human melanoma (Riehl and Tilgen 1982), in this study were demonstrated by FE to occur also in fish without any structural deviation. They are interpreted to be autophagic melanosome degrading vacuoles (Vielkind et al. $1971 \mathrm{a}, \mathrm{b}$; Sobel et al. 1975).

In FE replicas in particular, fish melanomas cannot be distinguished from those of humans. There are no differences in architecture and inner organization. Both melanoma in fish and in humans show cells with prominent dendritic processes, abundant melanosomes, and large, lobulated nuclei, which have numerous nuclear pores.

Acknowledgements. We thank Prof. F. Anders for many helpful suggestions and Prof. E. Paul and Dr. Said-Ali for critical reading of the manuscript. The perfect technical assistance of Mrs. H. Wahn is gratefully acknowledged. This work was supported by Deutsche Forschungsgemeinschaft through SFB 103 ,Zellenergetik und Zelldifferenzierung" and SFB 136 „Krebsforschung."

\section{References}

Anders A, Anders F (1978) Etiology of cancer as studied in the platyfish - swordtail system. Biochim Biophys Acta - Rev Cancer 516:61-95

Anders F, Diehl H, Schwab M, Anders A (1979) Contribution to an understanding of the cellular origin of melanoma in the GordonKosswig xiphophorine fish tumor system. Pigm Cell 4:142-149

Anders F, Schartl M, Scholl E (1981) Evaluation of environmental and hereditary factors in carcinogenesis, based on studies in $\mathrm{Xi}$ phophorus. In: Dawes CJ et al. (eds) Phyletic approaches to cancer. Japan Scientific Societies Press, Tokyo (pp 289-309)

Anders et al. (to be published) Xiphophorus as an in vivo model for studies on normal and defective control of oncogenes. Adv Cancer Res 42
Branton D, Bullivant S, Gilula NB, Karnovsky MJ, Moor H, Mühlethaler K, Northcote DH, Packer L, Satir B, Satir P, Speth V, Staehelin LA, Steere RL, Weinstein RS (1975) Freeze-etching nomenclature. Science 190:54-56

Breathnach AS (1971) An atlas of the ultrastructure of human skin. $\mathrm{J}$ and $\mathrm{A}$ Churchill, London

Breathnach AS (1973) Freeze-fracture replication of melanocytes and melanosomes. Br J Dermatol 89 (suppl 9):18

Breathnach AS, Gross M, Martin B (1973) Freeze-fracture replication of melanocytes and melanosomes. J Anat 116:303-320

Hudson CS, Rash JE, Graham WF (1979) Introduction to sample preparation for freeze-fracture. In: Rash JE, Hudson CS (eds) Freeze-fracture: methods, artifacts, and interpretations. Raven Press, New York (pp 1-10)

Hunter JJA, Zaynoun S, Paterson WD, Bleehan SS, Mackie R, Cochran AJ (1978) Cellular fine structure in the invasive nodules of different histogenetic types of malignant melanoma. Br J Dermatol 98:255-272

Lea PJ, Haberman HF, Pawlowski A, Menon IA (1976) The use of freeze-fracture and negative staining techniques to study the ultrastructure of melanosomes isolated from $\mathrm{B} 16$ melanoma. J Anat 121:1-5

Peracchia C, Mittler BS (1972) Fixation by means of glutaraldehyde hydrogen peroxide reaction products. J Cell Biol 53:234-238

Reynolds ES (1963) The use of lead citrate at high $\mathrm{pH}$ as an electronopaque stain in electron microscopy. J Cell Biol 17:208-212

Riehl R, Tilgen W (1982) Morphological aspects of human malignant skin tumors: A freeze-fracture study. J Cutan Pathol 9:225240

Romeis B (1968) Mikroskopische Technik. R Oldenbourg, München (pp 368-370)

Sobel HJ, Marquet E, Kallman KD, Corley GJ (1975) Melanomas in platy/swordtail hybrids. In: Ribelin WE, Migaki G (eds) The pathology of fishes. The University of Wisconsin Press, Madison (pp 945-981)

Tilgen W, Riehl R, Schroeter D (1981) Freeze-etching of human main platy/swordtail hybrids. In: Ribelin WE, Migaki G (eds) The

Vielkind J, Vielkind U, Anders F (1971 a) Melanotic and amelanotic melanomas in xiphophorin fish. Cancer Res 31:868-875

Vielkind J, Vielkind U, Anders F (1971 b) Electron microscopic studies on melanotic and amelanotic melanomas in xiphophorin fish. Z Krebsforsch 75:243-245

Weissenfels N, Schäfer D, Bretthauer R (1970) Über die Entartung der Makromelanophoren und den Einfluß des infiltrierenden Melanomwachstums auf die Muskulatur von Poeciliiden-Bastarden. Virchows Arch [Cell Pathol] 5:144-158

Zelickson AS (1967) Melanocyte, melanin granule and Langerhans cell. In: Zelickson AS (ed) Ultrastructure of normal and abnormal skin. Lea and Febiger, Philadelphia (pp 163-182)

Received March 3, 1983/Accepted September 18, 1983 